\title{
Electron microscopy of glial cells of the central nervous system in the crab Ucides cordatus
}

\section{S. Allodi and}

M. Taffarel
Departamento de Histologia e Embriologia, Instituto de Ciências Biomédicas, Universidade Federal do Rio de Janeiro, Rio de Janeiro, RJ, Brasil

\begin{abstract}
Correspondence

S. Allodi

Departamento de Histologia e

Embriologia, ICB, CCS, UFRJ

21941-590 Rio de Janeiro, RJ

Brasil

Fax: +55-21-287-4992

E-mail: sallodi@ chagas.biof.ufrj.br

Presented at the XIII Annual Meeting of the Federação de Sociedades de Biologia Experimental, Caxambu, MG, Brasil, August 26-29, 1998.

Research supported by CNPq, CAPES, FINEP, CEPEG/UFRJ, FUJB.

Received March 24, 1998

Accepted November 10, 1998

Invertebrate glial cells show a variety of morphologies depending on species and location. They have been classified according to relatively general morphological or functional criteria and also to their location. The present study was carried out to characterize the organization of glial cells and their processes in the zona fasciculata and in the protocerebral tract of the crab Ucides cordatus. We performed routine and cytochemical procedures for electron microscopy analysis. Semithin sections were observed at the light microscope. The Thiéry procedure indicated the presence of carbohydrates, particularly glycogen, in tissue and in cells. To better visualize the axonal ensheathment at the ultrastructural level, we employed a method to enhance the unsaturated fatty acids present in membranes. Our results showed that there are at least two types of glial cells in these nervous structures, a light one and a dark one. Most of the dark cell processes have been mentioned in the literature as extracellular matrix, but since they presented an enveloping membrane, glycogen and mitochondria intact and with different degrees of disruption - they were considered to be glial cells in the present study. We assume that they correspond to the perineurial cells on the basis of their location. The light cells must correspond to the periaxonal cells. Some characteristics of the axons such as their organization, ensheathment and subcellular structures are also described.
\end{abstract}

Invertebrate glial cells show a variety of morphologies depending on species and location and have been classified according to certain relatively general morphological or functional criteria and according to their position $(1,2)$.

In the crustacean nervous system there is good anatomical and physiological evidence for an insulating sheath with nodes, and rapid saltatory conduction (3). The sheath is composed of many laminae, each one containing cytoplasm and forming a seam. The axons can be wrapped simply by a single

\section{Key words}

- Glial cells

- Crustaceans

- Electron microscopy

- Cytochemistry

- U cides cordatus glial process or in groups, or by complex, tightly packed multiple layers resembling vertebrate myelin (4). However, there are important differences from vertebrate myelin. The most evident one is that the laminae do not connect to form a spiral and that the nuclei of the sheath cells lie inside the sheath (3).

The participation of neuroglia in the blood-brain barrier is another important function of glial cells $(5,6)$. Unlike the situation in higher vertebrates, where the blood-brain barrier is formed by tight junctions in endo- 
thelial cells lining central nervous system capillaries (7), in Crustacea the barrier is formed by glial perineurium surrounding the ganglia $(8,9)$. However, the most widely held belief about the functions of glial cells is that they interact metabolically with neurons, providing a trophic supply, exchanging metabolites and removing catabolites. This is supported by the presence of glycogen and of large numbers of vesicular and granular inclusions inside the glial cell cytoplasm (9).

The aim of the present study was to characterize the organization of glial cells and their processes by electron microscopy (EM) and correlate it with the extracellular matrix distribution in the protocerebral tract (PT) and in the zona fasciculata (ZF) of the crab Ucides cordatus. We carried out routine procedures (optic lobes were dissected and fixed by immersion in $2.5 \%$ glutaraldehyde and $4 \%$ paraformaldehyde in $0.1 \mathrm{M}$ cacodylate buffer, $\mathrm{pH} 7.3$, post-fixed in $1 \%$ osmium tetroxide and embedded in Epon) and the same procedure with the addition of $0.1 \%$ tannic acid in the fixative to stain the extracellular matrix (10); semithin sections stained with toluidine blue were observed at the light microscope level. We applied the method of Thiéry to identify glycogen (11),

Figure 1 - A, Electron micrograph of the protocerebral tract (PT) treated according to Angermüller and Fahimi (12). Observe that there is no relationship between the axon diameter and thickness of the ensheathment. Note vesicular structures (arrows) and mitochondria inside the axons (arrowheads). Also note the dark (stars) and light (asterisks) glial processes. Bar: $1.0 \mu \mathrm{m}$. B, Electron micrograph of the PT showing a light glial cell enveloping an axon (asterisk). Glial cell nucleus (N). Note that the outermost ensheathment of this axon is composed of a dark cell process (star). Bar: $0.6 \mu \mathrm{m}$.

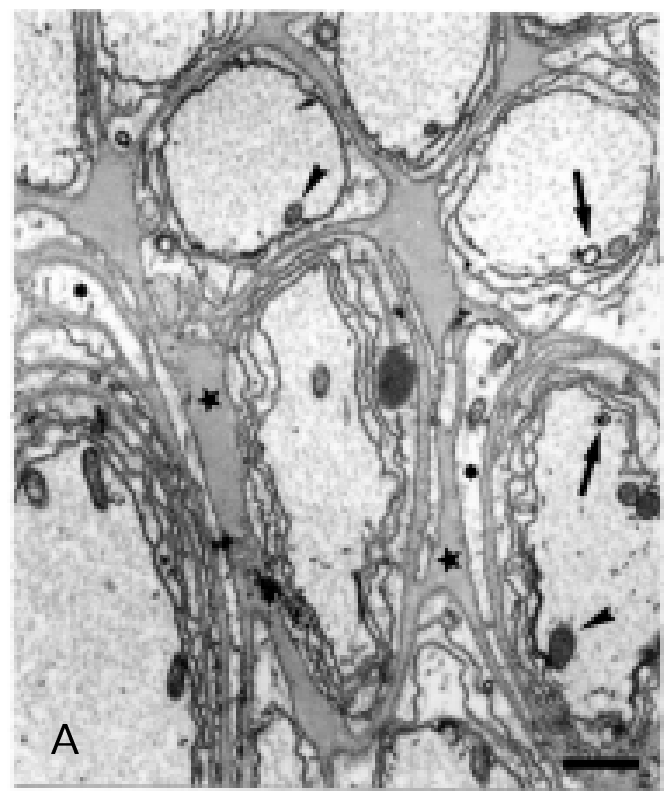

and the method of Angermüller and Fahimi (12) to demonstrate at the EM level unsaturated fatty acids such as those present in biological membranes for better visualization of the axonal ensheathment. The PT and the ZF were chosen because their structural elements are mostly axons and glial cells.

Toluidine blue-stained transversal semithin sections of the fascicles in the ZF showed axons of different diameters. Glial cell nuclei could be seen at the periphery and also among axon bundles. Some of them were very close to the axons. Surrounding the fascicles we observed a more intensely stained material which was continuous among fibers. In the PT a similar organization could be seen, i.e., projections departing from cell bodies continuous with an intensely stained material surrounding both the tract and the fibers. This dense material also enveloped the hemolymph vessels.

In routine and cytochemical preparations for EM, many aspects of the cytoarchitecture of these structures could be better visualized and are described as follows: a) axons - they confirmed our previous light microscopy observation that the differently sized axons were not organized in a regular pattern in the $\mathrm{ZF}$ or the PT, i.e., large axons, grouped or

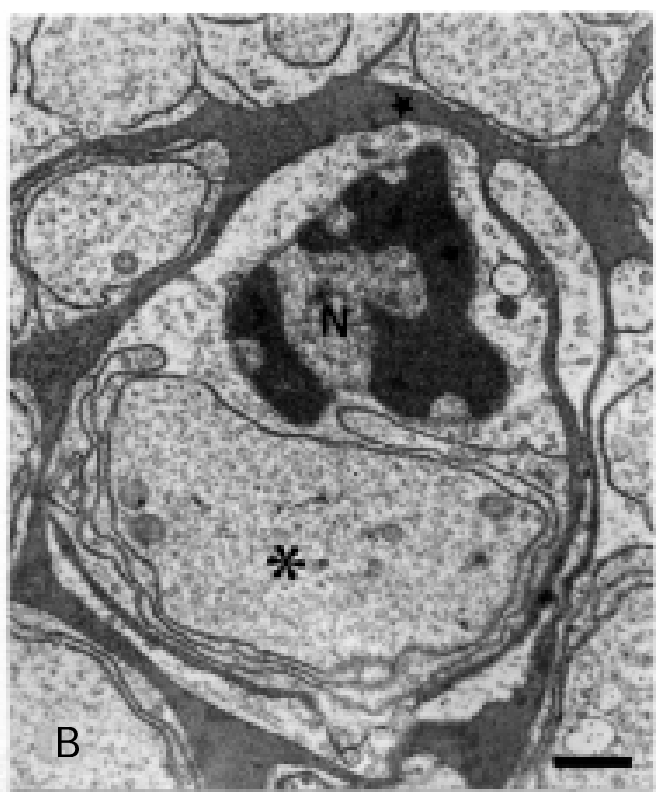


not, were dispersed among small ones, also grouped or not. Transversal sections of fibers exhibited differences in axonal ensheathment. We could not see any relationship between axon diameter and thickness of the wrappings. Groups of thinner fibers were occasionally circled by a glial process. These features were better visualized by the method of Angermüller and Fahimi, since the membranes resulted more electrondense. This method also revealed vesicular structures of different sizes inside the axons, probably originating from the endoplasmic reticulum. The cytoplasm of these axons presented many mitochondria, especially at the periphery, and fibrillar structures. Occasional microtubules could also be observed (Figure 1A). b) Glial cells - two types of processes could be noted surrounding the axons both in the ZF and in the PT: a light one and a dark one. Some axons were enveloped by alternating dark and light processes and others, only by light ones, which could be single or multiple. Some of the light processes were clearly associated with their cell nuclei which are located close to the axons and others had their nuclei among the fibers (Figure 1B). It is interesting to note that the innermost process enveloping each axon was always of the light type (Figure 1A and B). They contain microtubules, mitochondria and rosettes of glycogen aggregates, as confirmed by the method of Thiéry. The glycogen could be found dispersed in the cytoplasm, surrounded by a membrane, or among fibrillar material. Small vesicles could be occasionally seen (Figure 2A). Many peripheral cells presented their cytoplasm filled with positive material after the Thiéry procedure, especially in the ZF. Electrondense processes (dark processes) were seen along the periphery of the PT and intermingling among fibers and bundles as well as surrounding vessels. A similar aspect could be seen in the ZF. An interesting feature is that we could observe the same glycogen aggregates in this type of process. A closer observation revealed that they are mainly composed of fibrils with a smaller diameter than microtubules. These fibrils could be better observed when transversally sectioned (Figure 2A). In the ZF occasional mitochondria were immersed in these processes. Figure 2B shows that they present different degrees of disruption, which could
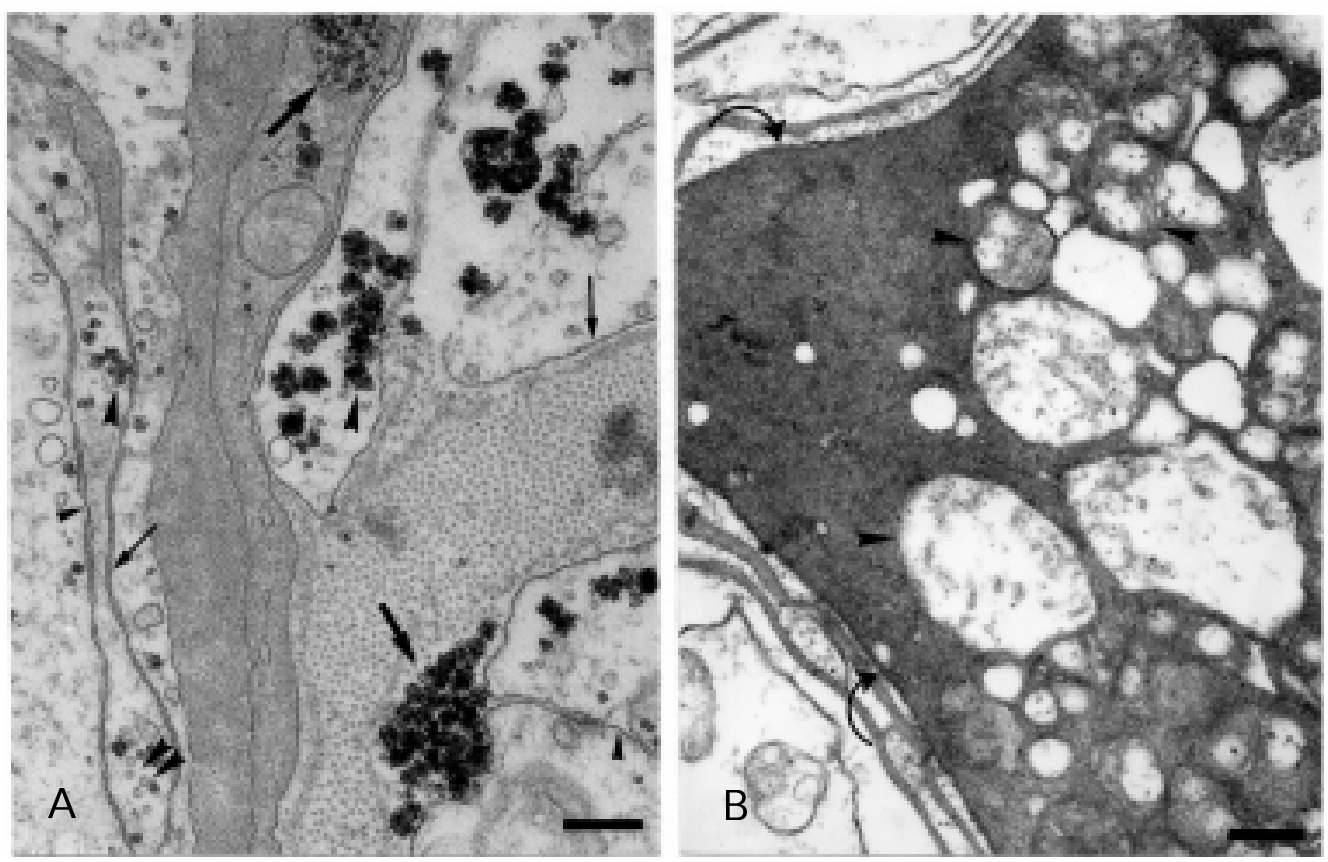

Figure 2 - A, Electron micrograph of the PT treated according to Thiéry showing glycogen aggregates in the cytoplasm of dark (large arrows) and light (large arrowheads) glial cell processes. Note that both the dark and the light processes clearly present a plasma membrane (thin arrows). Microtubules are observed in the light process (double small arrowheads) and also gap junctions (small arrowheads). Bar: $0.3 \mu \mathrm{m}$. B, Electron micrograph of the zona fasciculata treated according to Thiéry (11). The dark process displays mitochondrial profiles in different degrees of disruption (arrowheads). Observe a plasma membrane between the dark and light processes (curved arrows). Bar: 0.4 $\mu \mathrm{m}$. 
be better visualized at the periphery of the fascicles of the ZF and of the PT. Vesicles were seen in close proximity to them. We occasionally observed some membranous formations between light and dark processes. The dark processes presented a plasma membrane (Figure 2A). Between glial cells and axons and between glial cells there were gap junctions arranged along different extensions of the plasma membrane (Figure 2A) similar to those first reported by Cuadras and colleagues (13). Other types of junctions were not observed. By adding tannic acid to the fixative, collagen bundles could be identified in the outermost region of the PT and $\mathrm{ZF}$, but not near the dark processes.

It is known that in the vertebrate brain, glycogen, the form in which cells store glucose, is localized almost exclusively in astrocytes (7). In invertebrates (insects), Tsacopoulos and colleagues (14) demonstrated that glial cells around the photoreceptor neurons contain large amounts of this form of metabolic reserve and Wigglesworth (15) showed that glycogen is stored in large amounts in perineurial cells. Our results using the Thiéry method confirmed that it is suitable to study morphological aspects of glial cells in crustaceans, since it can be used as a label for these cells. The electronmicrographs permitted us to easily identify axons and glial cells in routine as well as in cytochemically treated material. Axons never presented glycogen and displayed many mitochondria and filamentous structures (Figure 1A). An interesting feature was that, unlike vertebrate axons, they presented few if any microtubules. All these features contrasted with those presented by glial cells. Routine and Thiéry methods showed that glycogen and also some mitochondria are present in the two types of processes (light and dark) described here. So, we assumed that these processes belong to two types of glial cells. Some investigators who worked with crustaceans have considered structures similar to the dark processes described in this work to be extracellular matrix, which they called connective tissue (16), or extracellular cement substance (1), or collagen layers (17). We disagree with at least part of this view. After an accurate observation, we have some arguments favoring the cellular nature of these structures: 1) they are surrounded by a plasma membrane, not always evident, 2) they exhibit glycogen granules among fibrillar constituents, which are considered to be a strictly intracellular reserve, 3) they present mitochondria and light vacuoles, and 4) cell bodies peripheral to the ZF and the PT, whose cytoplasm shows loosely packed fibrils among glycogen granules, display the same pattern as those seen in the dark processes.

If all these dark processes were considered to be extracellular matrix, this fact would be strikingly different from the vertebrate nervous system. As a matter of fact, our electron micrographs clearly demonstrated that there is not much space between axons and glial cells. Since the matrix is present, it may be restricted to small spaces among cells, between cells and vessels and between cells and the outermost limits of the nervous structure.

On the basis of these considerations, we propose that the light and dark processes correspond to the glial cells called periaxonal and perineurial glia, respectively (18), since the former were continuous with nuclei close to axons and the latter were continuous with a cell body located at the periphery of both structures studied by us.

Regarding the mitochondria in the perineurial glial processes, our electron micrographs showed that they present different degrees of disruption when they are located at the periphery of the fascicles of the ZF and of the PT and that this disruption may be absent in the inner aspects of these nervous structures.

Taken together, the observations concerning the perineurial glial projections surrounding axons, fascicles, tract and hemo- 
lymph vessels led us to propose that the dark material results from a maturation process where the synthesis and accumulation of fibers and glycogen inside the glial cells cytoplasm occur in a manner that in some aspects resembles that of keratinization in the skin of vertebrates (19). As a result of this maturation process, the organelles present in these cells must progressively disappear as clearly shown in the present study for mitochondria. Our proposal is that these glial cell processes with a quite homogeneous dense material mature to act as an insulating envelope that can be compared to the myelin sheath of vertebrates. This highly specialized glial wrapping may be one of the features responsible for the high conduction velocities of nerve impulses in crustaceans $(3,20)$.

\section{Acknowledgments}

We are grateful to Dr. Ana Maria Blanco Martinez for helpful discussions and to Ms. Nanci Barroso Gonçalves da Silva for technical assistance.

\section{References}

1. Hámori J \& Horridge GA (1966). The lobster optic lamina. IV. Glial cells. J ournal of Cell Science, 1: 275-280.

2. Radojcic T\& Pentreath VW (1979). Invertebrate glia. Progress in Neurobiology, 12: 115-179.

3. Heuser JE \& Doggenweiler CF (1966). The fine structural organization of nerve fibers, sheaths, and glial cells in the prawn Palaemonetes vulgaris. J ournal of Cell Biology, 30: 381-403.

4. Bullock TH, Orkand R \& Grinnel A (1977). Introduction to Nervous Systems. W.H. Freeman and Co., San Francisco, London.

5. Abbott NJ, Bundgaard M \& Cserr HF (1986). Comparative physiology. Annals of the New York Academy of Sciences, 481: 20-41.

6. Abbott NJ (1995). Morphology of nonmammalian glial cells: functional implications. In: Kettermann H \& Ransom B (Editors), Neuroglia. Oxford University Press, Oxford and New York.

7. Peters A, Palay SL \& Webster H de F (1991). In: The Fine Structure of the Nervous System: Neurons and their Supporting Cells. Oxford University Press, Oxford, New York.
8. Abbott NJ (1972). Access of ferritin to the interstitial space of Carcinus brain from intracerebral blood vessels. Tissue Cell, 4: 99-104.

9. Pentreath VW (1987). Functions of invertebrate glia. In: Ali MA (Editor), Nervous System in Invertebrates. Plenum Press, New York and London, 61-103.

10. Sanes J R (1989). Extracellular matrix molecules that influence neural development. Annual Review of Neuroscience, 12: 491516.

11. Thiéry J P \& Rambourg A (1974). Cytochimie des polysaccharides. J ournal de Microscopie, 21: 225-232.

12. Angermüller $S \&$ Fahimi HD (1982). Imidazole-buffered osmium tetroxide: an excellent stain for visualization of lipids in transmission electron microscopy. Histochemical J ournal, 14: 823-835.

13. Cuadras J, Martin G, Czternasty G \& Bruner J (1985). Gap-like junctions between neuron cell bodies and glial cells of crayfish. Brain Research, 326: 149-151.

14. Tsacopoulos M, Coles JA \& Van Der Werve G (1987). The supply of metabolic substrate from glia to photoreceptors in the retina of the honeybee drone. J ournal de Phisiologie, 82: 279-287.

15. Wigglesworth VB (1960). The nutrition of the central nervous system in the cockroach Periplaneta americana $\mathrm{L}$ : the role of the perineurium and glial cells in the mobilization of reserves. J ournal of Experimental Biology, 37: 500-512.

16. Shivers RR \& Brightman MW (1976). Trans-glial channels in ventral nerve roots of crayfish. J ournal of Comparative Neurology, 167: 1-26.

17. Parnas I, Shahrabany-Baranes $O$, Feinstein N, Grant P, Adelsberger H \& Dudel J (1998). Changes in the ultrastructure of surviving distal segments of severed axons of the rock lobster. J ournal of Experimental Biology, 201: 779-791.

18. Grossfeld RM (1991). Axon-glia exchange of macromolecules. In: Abbot NJ (Editor), Glial-Neuronal Interaction. New York Academy of Sciences, New York, 319330.

19. Gartner LP \& Hiatt J L (1997). Color Textbook of Histology. W.B. Saunders Company, Philadelphia.

20. Pentreath VW (1989). Invertebrate glia cells. Comparative Biochemistry and Physiology, 93A: 77-83. 